\title{
Challenges in Deciphering New Mechanisms in Cellular Biology
}

\section{Anja Nohe*}

Department of Biological Sciences, University of Delaware, USA

*Corresponding author: Anja Nohe, Department of Biological Sciences, University of Delaware, USA, Tel: (302) 831-2959; E-mail: anjanohe@UDel.Edu

Received date: 30 Jan, 2015, Accepted date: 2 Feb, 2015 Published date: 9 Feb, 2015

Copyright: (c) 2015 Anja Nohe. This is an open-access article distributed under the terms of the Creative Commons Attribution License, which permits unrestricted use, distribution, and reproduction in any medium, provided the original author and source are credited.

\section{Editorial}

A few decades ago scientific progress in biology was slow. The identification of new mechanisms on the cellular level was restricted to observations in embedded or fixed samples isolated and processed using complicated protocols. This pattern changed with the development of new imaging techniques that allowed the observation of cellular dynamics at fast time ranges from the nanosecond to millisecond. The development of kits allowed for rapid sample processing. New probes for labelling and high resolution microscopy completely changed the way of scientific exploration. Things that were thought of as unfeasible were all of a sudden possible. These advances combined with the growth in areas of genomics and proteomics research allowed for the rapid production of mass data and articles that describe observations at fast and slow timescales. Additionally, data are available on various length scales, from nano-meters to $\mathrm{cm}$. These observations are published on a daily basis. The new open access rules and regulations for researchers funded by federal research institutions such as the National Institute of Health allows the dissemination of the new data over the internet conveniently.

However, for researchers working in their specific area, it is challenging to keep up with the current literature, especially data interpretation is often difficult. Although scientists can investigate and describe cellular processes in more detail, questions about cellular mechanisms remain often unanswered. Especially more detailed mechanisms of how the cell functions and how it can be regulated must be determined. New ideas and hypotheses need to be formulated and investigated in a more scientific manner. This requires a newer generation of biologists skilled in critical thinking that are familiar with modern approaches of data analysis, statistics and quantitative biology. In addition the newer generation should be able to utilize and design computational models. The new area of system biology is a starting point and melting pot for this development. Teams of interdisciplinary trained biologists, chemists, physicists and engineers merge together to form a new microcosm, in which these challenges can be addressed. The networking of scientists from varying disciplines allows for the formulation of computational models that may test hypotheses and formulate new dogmas and mechanisms. These can then be verified experimentally. These approaches leads to new models that envision cellular outcomes such as lineage determination and cell proliferation. Using systems biology approaches to predict signalling networks, protein localization and cellular response at various timescales will drive research into the new millennium.

However, in order to secure this line of research, funding interdisciplinary high risk projects is fundamental. Research that explores new avenues and bridges system biology with biophysics and experimental biology needs reviewers and review panels accustomed with a wide variety of approaches. Obviously producing preliminary data for the modelling and experimental setups to define new mechanisms is very time consuming and funding for high risk projects is currently lacking in many areas. And obviously should be one of the most important priorities in today's science.

\section{Acknowledgement}

Gina Caridi and the RO1 funding: 5R01AR064243-02, NIAMS 